\title{
IMPACTO DA CEVA NO SABOR E COLORAÇÃO DE FILÉS DO PEIXE MATRINXÃ SELVAGEM NA BACIA AMAZÔNICA
}

\author{
IMPACTO DE LA CEBA EN EL SABOR Y COLORACIÓN DE LOS FILETES DE \\ PEZ MATRINXÃ SILVESTRE EN LA CUENCA AMAZÓNICA
IMPACT OF CHUM IN FLAVOR AND COLORING OF FILLETS OF WILD FISH MATRINXÃS IN THE AMAZON BASIN

\author{
LILIANE MATOS S, ${ }^{1,3}$ M.Sc, JOÃO OTÁVIO SILVAS, ${ }^{2}$ ALINE TESK, ${ }^{2}$ LUCÉLIA CARVALHO N, ${ }^{1,2,3}$ Dra. \\ ${ }^{1}$ Programa de Pós-Graduação em Ecologia e Conservação da Biodiversidade. Universidade Federal de Mato Grosso-UFMT, \\ Campus Universitário de Cuiabá, 78060-900. Cuiabá, MT, Brasil. \\ ${ }^{2}$ Instituto de Ciências Naturais, Humanas e Sociais - UFMT, Campus Universitário de Sinop, 78557-267. Sinop, MT, Brasil. \\ ${ }^{3}$ Programa de Pós-Graduação em Ciências Ambientais. Universidade Federal de Mato Grosso-UFMT.Campus \\ Universitário de Sinop, 78557-267. Sinop, MT, Brasil.
}

Palavras-chave:

Brycon falcatus, análise sensorial, rio Teles Pires, rio Cristalino, soja.

Key words:

Brycon falcatus, sensory analysis, Teles Pires river, Cristalino river, soy bean.

\section{Resumo}

Atualmente, na bacia do rio Teles Pires, norte de Mato Grosso, uma das técnicas mais utilizadas pelos pescadores para captura do peixe matrinxã é a ceva. A ceva é a oferta de suplemento alimentar, neste caso, grãos de soja in natura colocados em lugar determinado para atrair principalmente os peixes onívoros. O objetivo deste estudo foi avaliar se existe diferença no sabor e na cor de matrinxãs selvagens (Brycon falcatus) capturados com a presença de ceva e sem a presença de ceva. Os peixes foram coletados na estação seca do ano de 2013, no rio Teles Pires, com ceva, e no rio Cristalino, sem ceva. Foram anotados comprimento padrão e peso dos peixes e retirados os filés, que foram congelados. Após descongelamento, os filés foram individualmente cozidos em forno micro-ondas. Para avaliar se havia diferença no sabor dos filés de matrinxã foi aplicado o método triangular. Para determinar a coloração do filé, foi utilizado o método modificado da escala colorimétrica Salmofan@. Na análise sensorial, realizada com 42 provadores foi detectada diferença significativa no sabor, com um nível de probabilidade de $0,5 \%$. E, visualmente também foi detectada diferença na coloração dos filés, tendo os peixes do rio Cristalino maior tendência a tons salmão. Os resultados indicaram que a presença de ceva está alterando o sabor e coloração do filé do peixe matrinxã.
INFORMACIÓN Recibido: 21-04-2015: Aceptado: 23-07-2015. Correspondencia autor: lilistedile@hotmail.com

\begin{abstract}
Currently, in the watershed of the River Teles Pires, southern amazon (Mato Grosso), the most widely used technic to catch fishes belonging to "mantrinxa" is baiting. Most fisherman are using soybean in well-defined places to attract omnivorous fishes. The aim of our study was to evaluate potential savour and colour differences of wild matrinxãs (Brycon falcatus) caught with or without feeding. Fish were caught in 2013 during the dry period in the Teles Pires River while attracting them with soybean and in the river Cristalino, without baiting them. Both size and weight of each fish were noted and fillets were removed to be cooked using a microwave. We used a triangular test to assess potential differences in flesh taste. We also used a modified colorimetric Salmofan@ $\odot$ method to determine coloration of each fillet. Forty-two testers were selected for the sensorial (gustative) analyse and a significant difference was found regarding taste. We also detected differences in flesh coloration, with fish from the Cristalino River presenting colouration closer to salmon shades. Results indicated that baiting fish modify both colour and savour of matrinxãs fillets.
\end{abstract}


Palabras Clave:

Brycon falcatus, análisis sensorial, río Teles Pires, río Cristalino, soya.

\begin{abstract}
Resumen
Actualmente, en la cuenca del río Teles Pires, norte de Mato Grosso, una de las técnicas más utilizadas por los pescadores para captura de matrinxã es la ceba. La ceba es una oferta de suplemento alimentar, en este caso, granos de soja in natura colocados en un lugar determinado para atraer principalmente a los peces omnívoros. El objetivo de este estudio fue evaluar si existe diferencia en el sabor y el color de matrinxãs silvestres (Brycon falcatus) capturados mediante la ceba y sin la presencia de ceba. Los peces fueron colectados en la estación seca del año 2013, en el río Teles Pires, con ceba, y en el río Cristalino, sin ceba. Se anotó longitud y peso de los peces y de los de los filetes, que fueron luego congelados. Después del descongelamiento, los filetes fueron individualmente cocidos en homo micro-ondas. Para evaluar si había diferencia en el sabor de los filetes se aplicó el test triangular. Para determinar la coloración del filete, se utilizó el método modificado de la escala colorimétrica Salmofan@C. En el análisis sensorial, realizado con 42 catadores se detectó diferencia significativa en el sabor, con un nivel de probabilidad de $0,5 \%$. Visualmente también fue detectada diferencia en la coloración de los filetes, siendo los peces del río Cristalino los que presentaron mayor tendencia a tonos de salmón. Los resultados indican que la presencia de la ceba está alterando el sabor y la coloración del filete de matrinxã.
\end{abstract}

\section{Introdução}

Atualmente, na bacia do rio Teles Pires, no norte de Mato Grosso, uma das técnicas mais utilizadas pelos pescadores para captura de matrinxãs, é a ceva, definida como alimentos (soja, milho, mandioca) colocados em lugar determinado para atrair principalmente os peixes onívoros. Estudos sobre o efeito da oferta de suplemento alimentar (ceva), no padrão de coloração e sabor de filés de peixes selvagens são escassos. 0 salmão selvagem e de cultivo comparações entre as propriedades organolépticas da carne (SYLVIA et al, 1995; FARMER et al., 2000; JOHNSTON et al., 2006), indicaram diferenças no sabor e coloração, entretanto, salmão de cultivo com dietas balanceadas são tão aceitáveis quanto os selvagens para consumo. Em estudo sobre a coloração do filé de piracanjuba (Brycon orbignyanus) selvagem e de cultivo, foi constatado que a coloração vermelha foi mais intensa nos filés dos peixes selvagens devido sua alimentação rica em carotenoides (SANTAMARIA e ANTUNES, 1998/1999).

Brycon falcatus (MÜLLER e TROSCHEL, 1844) é uma espécie migradora, popularmente conhecida como matrinxã, pertencente à subfamília Bryconinae, e ocorre nas bacias hidrográficas das Guianas, Suriname, Amazonas, Orinoco e Araguaia-Tocantins (LIMA, 2003). Estudos sobre a dieta de B. falcatus na bacia amazônica foram encontrados como itens alimentares: vegetais durante a estação chuvosa, artrópodes na estação seca, e folhas, flores, crustáceos e peixes nas duas estações (ALBRECHT et al., 2009; BLANCO-PARRA e BEJARANO-RODRÍGUEZ, 2006; MATOS, 2014). Algumas espécies de Brycon possuem a coloração da carne laranja-avermelhada devido à deposição de pigmentos carotenoides provenientes de alimento natural (camarões, caranguejos), conferindo um agradável aspecto visual e, provavelmente, um maior valor de mercado (SANTAMARIA e ANTUNES, 1998/1999).

Com a implantação de cevas no habitat natural, o comportamento alimentar do matrinxã vem sofrendo alterações (MATOS, 2014), o que reflete na composição química e provoca alterações nas propriedades organolépticas da carne. Segundo KUBITZA (2000), a maioria dos grãos usados nas rações não alteram o sabor e odor dos filés de maneira detectável pelos consumidores, mas podem causar diferenças na coloração e textura da carne, dependendo do tipo de ingrediente e do nível de inclusão na dieta. No rio Teles Pires, verificou-se que a dieta do matrinxã é cerca de $90 \%$ baseada em soja in natura (MATOS, 2014), provavelmente proveniente da ceva. E isto, está ocasionando grande deposição de gordura no músculo (MATOS, 2014). Existe a possibilidade da dieta dos matrinxãs estar afetando a coloração do filé, pois estes peixes estão deixando de se alimentarem dos itens que possuem carotenoides, como por exemplo os crustáceos (OGAWA E MAIA, 1999). Os consumidores já percebem a diferença no sabor e coloração dos peixes, pois pescado oriundo do rio Teles Pires já é qualificado regionalmente como "peixe de ceva". O objetivo deste estudo foi verificar se existe diferença no sabor e na cor do filé do peixe matrinxã de locais com ceva e sem ceva, utilizando como ferramenta a análise sensorial. Considerando que alteração da dieta do peixe pode alterar seu sabor e coloração, levantamos as seguintes hipóteses: a) os provadores da análise sensorial detectarão diferença no sabor dos filés dos matrinxãs oriundos do rio Cristalino e do rio Teles Pires; b) matrinxãs oriundos do rio Cristalino apresentarão filés com a coloração salmão mais intensa do que os do rio Teles Pires. 


\section{Material e métodos}

Área de estudo: A bacia do rio Teles Pires está inserida no norte do Estado de Mato Grosso e na Amazônia Legal, formando um dos principais contribuintes do rio Tapajós. As principais atividades econômicas no entorno desta bacia tem sido a exploração de madeira, extração de ouro, pecuária e agricultura (principalmente soja e milho), o que tem levado a um grande comprometimento dos recursos hídricos (BARTHEM e GOULDING, 1997). A coleta de peixes nesse rio foi realizada em um trecho

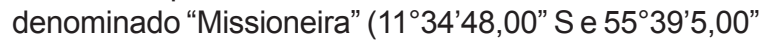
W) (Fig. 1), com alta densidade de cevas de soja in natura, sendo aproximadamente uma ceva para cada $100 \mathrm{~m}$ de rio. A soja in natura é ofertada ilegalmente aos peixes hidratada em água ou na forma natural (seca). As cevas podem ser feitas de várias formas: 1) com tambores plásticos de 50 e $200 \mathrm{~L}$ perfurados para que a soja saia de forma contínua submersos na água e fixados próximos a trapiches; 2) em canos de PVC de $200 \mathrm{~mm}$ e $6 \mathrm{~m}$ de comprimento, perfurados, dispostos no leito do rio, e também com sacos de ráfia perfurados, submersos na água. Para comparação do sabor e coloração dos filés dos peixes capturados em cevas, foram coletados exemplares da mesma espécie no rio Cristalino (Fig. 1), onde não existem cevas, sendo considerado como tratamento controle. O rio Cristalino $\left(9^{\circ} 32^{\prime} 47,00^{\prime \prime} S\right.$ e $\left.55^{\circ} 47^{\prime} 38,00^{\prime \prime} \mathrm{W}\right)$ está inserido, em sua maior parte, no Parque Estadual do Cristalino - PEC - MT, e toda área do entorno desta bacia está legalmente protegida contra o desmatamento e a ocupação humana.

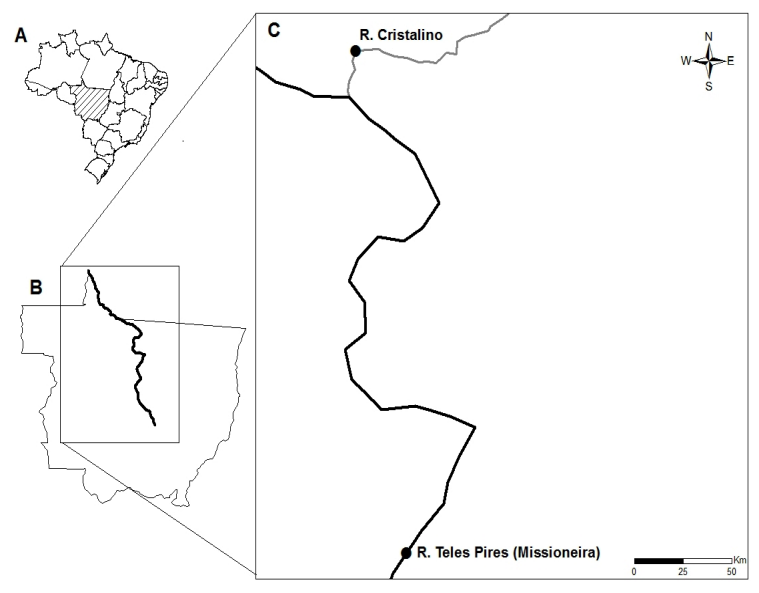

Figura 1. Área de estudo, mostrando os rios de coleta, Teles Pires e Cristalino, estado de Mato Grosso, Brasil.

Coletas de peixes e biometria: As coletas dos peixes foram na estação seca do ano de 2013, no rio Teles Pires (com ceva), e no rio Cristalino (sem ceva). Para as capturas, foram utilizadas varas com molinete e isca artificial. Após as capturas, os 18 peixes coletados, nove em cada local, foram anestesiados com Eugenol ${ }^{\circledR}$ (VIDAL et al., 2008), depois abatidos e em seguida, submersos em gelo. Os exemplares foram levados para o laboratório do Acervo Biológico Amazônia Meridional - ABAM na Universidade Federal de Mato Grosso - UFMT, onde foram coletados dados de biometria, comprimento total (CT), comprimento padrão (CP) e peso total (PT). Na sequência, foram retirados os filés de cada exemplar com instrumentos cirúrgicos de aço inoxidável (RIBEIRO E TOBINAGA, 2002), e estocados a $-20^{\circ} \mathrm{C}$ para posterior análise sensorial. Espécimes testemunhos do peixe matrinxã foram depositados no museu da Universidade Estadual de Campinas UNICAMP, tombo $n^{\circ}$ ZUEC 9190.

Análise Sensorial: Para análise sensorial, os filés foram descongelados, a pele foi retirada, e foram individualmente cozidos em forno micro-ondas durante 8 minutos, não sendo adicionado sal ou nenhum tempero para que não houvesse interferência no sabor. Foram mantidos em caixas térmicas até a degustação, onde foram seccionados em amostras de aproximadamente cinco gramas. Com a finalidade de "lavar" as células gustativas da boca, no intervalo entre a degustação entre as amostras, cada provador ingeriu um biscoito água e sal e água, simulando um "bochecho". Os 42 provadores receberam e assinaram o Termo de Consentimento Livre e Esclarecido-TCLE, onde ficaram esclarecidos os objetivos, riscos e benefícios desta pesquisa.

Para avaliar os efeitos da ceva no sabor dos filés de matrinxã, foi aplicado o método triangular, capaz de detectar pequenas diferenças entre amostras. Este método é utilizado para detectar pequenas diferenças, não avaliando o grau, nem caracterizando os atributos responsáveis pela diferença (DUTCOSKY, 1996). No Laboratório de Tecnologia de Alimentos da UFMT foram apresentadas simultaneamente três amostras codificadas com três dígitos e instruções de que, duas amostras eram iguais e uma diferente e uma ficha para assinalar a resposta, solicitando-se que provassem e examinassem cada amostra da esquerda para a direita, e selecionassem a amostra diferente (escolha forçada) (ABNT, 1993). A probabilidade de acertos é $p$ $=1 / 3$. A interpretação do resultado se baseia no número total de julgamentos versus o número de julgamentos corretos (ROESSLER et al, 1978). As amostras foram apresentadas casualizadas, nas permutações distintas: $A A B, B A A, A B A, A B B, B B A$ e $B A B$. A utilização de pares de amostras idênticas serviu para avaliar o "efeito placebo". E a utilização de amostras diferentes, alterando a ordem das mesmas, serviu para minimizar o erro de tendência posicional.

Análise da coloração: Para determinar a coloração do filé, foi utilizado o método modificado da escala 
colorimétrica Salmofan@, padronizando local, horário e luminosidade (ALFNES et al, 2006). Os filés foram descongelados em refrigerador na temperatura de $5^{\circ} \mathrm{C}$, e expostos à temperatura ambiente durante 30 minutos. Logo após, foram realizadas as imagens, sempre pelo mesmo observador, que fotografou (Canon Powershot G10 Digital 14.7MP) e anotou a coloração correspondente de cada amostra na escala colorimétrica (Fig. 2).

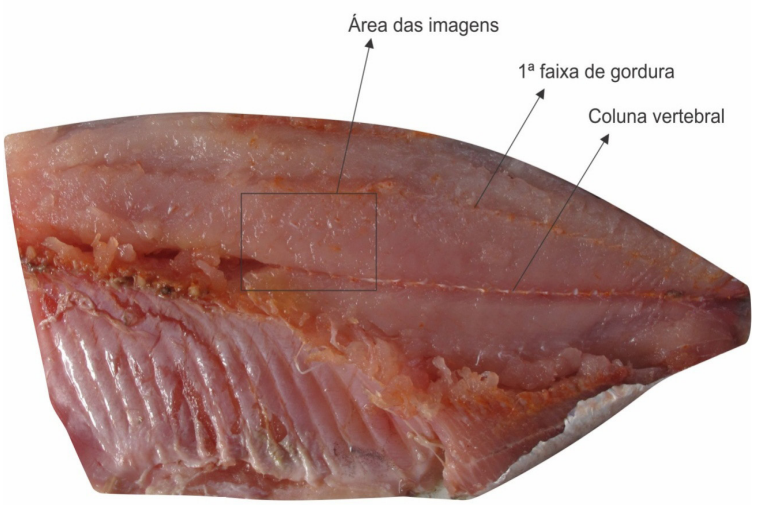

Figura 2. Filé de matrinxã Brycon falcatus utilizado na análise de coloração. Retângulo indica o local onde foram efetuadas as imagens dos filés.

Análise estatística: Para verificar a existência de diferença no sabor dos filés na análise sensorial, a estatística do método triangular se baseou no número mínimo de seleções corretas comparadas ao número de julgamentos totais, segundo ROESSLER et al. (1978) $(P<0,05)$.

\section{Resultados}

Os valores de comprimento padrão, comprimento total e peso dos exemplares do rio Teles Pires e rio Cristalino constam na Tabela 1.

Tabela 1.Médias e amplitudes do comprimento total (CT), comprimento padrão (CP) e peso dos exemplares de Brycon falcatus coletados em rios da bacia do Teles Pires.

\begin{tabular}{ccccc}
\hline Rios & $\mathbf{N}$ & $\mathbf{C T}(\mathbf{c m})$ & $\mathbf{C P}(\mathbf{c m})$ & Peso $\mathbf{( k g )}$ \\
\hline Cristalino & \multirow{2}{*}{9} & 46,00 & 34,25 & 1,64 \\
& & $(42,00-50,00)$ & $(27,50-41,00)$ & $(0,75-2,53)$ \\
Teles Pires & 9 & 43,50 & 35,00 & 1,88 \\
& & $(36,00-51,00)$ & $(29,00-41,00)$ & $(0,93-2,83)$ \\
\hline
\end{tabular}

Dos 42 julgadores, 23 selecionaram adequadamente a amostra diferente, então segundo (ROESSLER et al., 1978) as amostras diferiram significativamente $(P<$ $0,05)$. Dessa forma, foi detectada diferença sensorial quanto ao atributo sabor, entre o filé de matrinxã do rio Cristalino (sem cevas) e do rio Teles Pires no trecho denominado Missioneira (com cevas).
Os filés dos matrinxãs coletados no rio Teles Pires (Fig. 3, A ao I), apresentaram pouca pigmentação, tendendo à cor amarela, visto que, comparados à escala colorimétrica Salmofan $($, apresentaram tonalidade mais clara, estando próximos a tonalidade 20. A coloração dos filés dos matrinxãs coletados no rio Cristalino (Fig. 3, J ao R), apresentou tons salmão, e quando comparados à escala colorimétrica Salmofan@, apresentaram tonalidade entre o 24 e 26. Os filés L e M de exemplares coletados no rio Cristalino apresentaram coloração diferente da tonalidade salmão; estes exemplares foram os menores capturados, com comprimento padrão entre 27,50 e $32,50 \mathrm{~cm}$.

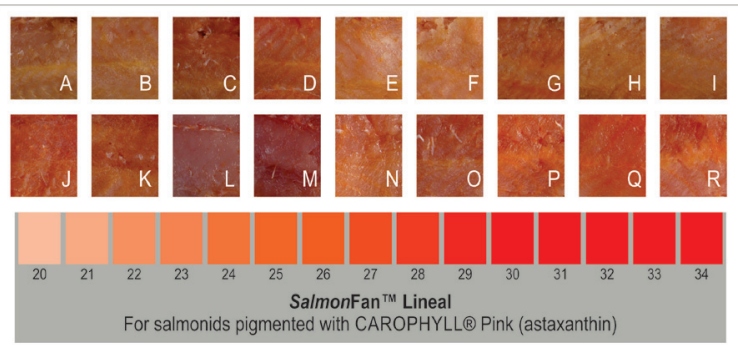

Figura 3. Filés de matrinxã Brycon falcatus utilizados para análise da coloração pelo método da escala colorimétrica Salmofan@ $\odot$. Amostras de A-I oriundas de indivíduos coletados no rio Teles Pires. Amostras de J-R oriundas do rio Cristalino.

\section{Discussão}

Neste estudo foi analisado se havia diferença entre as amostras de filé, e não qual amostra era mais saborosa, pois a escolha no sabor é muito peculiar, envolvendo a preferência pessoal por vários atributos. Os provadores encontraram diferença significativa entre os matrinxãs coletados em diferentes rios, demonstrando que existe algum fator que está influenciando seu sabor. Um estudo com truta arco-íris (Salmo gairdneri) que substituiu na dieta o óleo de arenque pela gordura suína, não encontrou diferença significativa nas características organolépticas da carne (BOGGIO et al., 1985). Em uma pesquisa com salmão (Salmo salar) com diferentes fontes lipídicas na dieta (óleos de soja, canola e capelin), os peixes alimentados com óleo de soja apresentaram alteração no sabor (THOMASSEN e ROSJO, 1989). MATOS (2014) analisaram o percentual de gordura no filé de indivíduos coletados em locais com ceva do rio Teles Pires e em locais sem ceva no rio Cristalino, encontrando que o percentual de gordura nos filés dos matrinxãs de locais de ceva foram significativamente maior. MATOS (2014) também analisou a dieta natural destes exemplares de matrinxã, onde foi constatado que a soja in natura foi o item mais importante (90\%) na dieta dos exemplares do rio Teles Pires, e os indivíduos 
coletados no rio Cristalino apresentaram uma dieta diversificada, tendo como principais itens: folhas $(9,6 \%)$, crustáceos $(8,5 \%)$, peixes $(6,3 \%)$ e sementes nativas (3\%). Desta forma, a diferença no sabor dos filés de matrinxãs pode estar associada à alteração da dieta natural no rio Teles Pires por soja in natura contida nas cevas (MATOS, 2014). Fato parecido foi relatado por SILVA et al. (2013), em um estudo com o peixe híbrido tambatinga, onde encontraram que a inserção de resíduos da agricultura (grãos de soja e milho) afetou negativamente o sabor dos filés. Então, podemos inferir que a alteração na dieta acarretou um aumento no percentual de gordura do filé, podendo assim, de acordo com as preferências de cada provador, conferir um sabor mais suculento ou rançoso ao filé.

Na comparação da coloração dos filés de matrinxãs, os filés Le M dos locais sem cevas, apresentaram coloração diferente da tonalidade salmão. Porém, os exemplares apresentaram tamanhos menores aos de primeira maturação que é de $35,00 \mathrm{~cm}$ conforme legislação ambiental vigente. Não existe estudo com Brycon apontando se a maturação sexual afeta a pigmentação do músculo. Mas nos salmonídeos, o processo de maturação sexual envolve mudanças significativas no metabolismo dos carotenoides, havendo redução drástica da quantidade dos carotenoides no músculo dos peixes alterando sua coloração (CHOUBERT e BLANC, 1993).

SANTAMARIA e ANTUNES (1998/1999) fizeram um estudo com Brycon orbignyanus selvagens e de cativeiro, e encontraram diferença na coloração da carne dos peixes selvagens, que apresentou coloração salmão devido sua alimentação rica em carotenoides. Em observação visual da coloração dos filés dos $B$. falcatus, pode-se notar a diferença na coloração dos filés de exemplares coletados no rio Teles Pires, que possuem como principal item alimentar soja in natura (MATOS, 2014), apresentando coloração tendendo ao amarelo. Os filés de peixes oriundos do rio Cristalino, que possuem uma dieta natural composta também por crustáceos, que são fonte de carotenoides (OGAWA E MAIA, 1999), apresentaram coloração tendendo ao salmão. Desta forma, podemos inferir que a diferença visual da coloração dos filés de matrinxã é devido à alteração da dieta.

\section{Conclusões}

Conclui-se que existe diferença no sabor e coloração entre os filés dos peixes matrinxãs coletados no rio Teles Pires, local onde há oferta suplementar de alimento de soja in natura, e no rio Cristalino, sem oferta de suplemento alimentar. Considerando que já existe legislação no estado de Mato Grosso que proíbe o uso de cevas no leito dos rios, as informações dos efeitos da ceva no matrinxã poderão ser utilizadas em políticas públicas, visando à integridade do peixe Brycon falcatus.

\begin{abstract}
Agradecimentos.
À Jéssika Tizuka, Daniela Rocha, Cleunice Mendes e Tatiane I. S. dos Santos, pelo auxílio nas atividades de laboratório. À Marcos Beckmann pelo auxilio nas coletas. Esta pesquisa foi realizada com o apoio da Secretaria Municipal de Meio Ambiente e Desenvolvimento Sustentável de Sinop / MT e Fundação de Amparo à Pesquisa do Estado de Mato Grosso - FAPEMAT edital 005 / 2012. A licença de coleta concedida pelo ICMBio (18924-1). LSM recebeu apoio financeiro da Coordenação de Aperfeiçoamento de Pessoal de Nível Superior - CAPES. Esta é a publicação número 49 da série técnica do Núcleo de Estudos da Biodiversidade da Amazônia Mato-Grossense - NEBAM.
\end{abstract}

\section{Referências}

ALBRECHT, M.P.; CARAMASCHI,E.P.; HORN, M.H. 2009. Population responses of two omnivorous fish species to impoundment of a Brazilian tropical river. Hydrobiologia 627:181-193.

ALFNES, F.; GUTTORMSEN, A. G.; STEINE, G.; KOLSTAD, K. 2006. Consumers' willingness to pay for the color of salmon: a choice experiment with real economic incentives. American Journal Agricultural Economics 88 (4):10501061.

ABNT - ASSOCIAÇÃO BRASILEIRA DE NORMAS TÉCNICAS. 1993. NBR 12995: Teste triangular em análise sensorial de alimentos e bebidas. Rio de Janeiro.

BARTHEM, R.; GOULDING, M. 1997. Os bagres balizadores: ecologia, migração e conservação de peixes amazônicos. Tefé, AM: Sociedade Civil Mamirauá. Brasília.

BLANCO-PARRA, M.D.P.; BEJARANO-RODRÍGUEZ, I. 2006. Alimentación y reproducción de las principales especies ícticas del río Mesay durante el período de "aguas altas". Revista de Biología Tropical 54 (3):853-859. 
BOGGIO, S.M.; HARDY, R.W.; BABBITT, J.K.; BRANNON, E.L. 1985. The influence of dietary lipid source and alpha-tocopheryl acetate level on product quality of rainbow trout (Salmo gairdneri). Aquaculture 51:13-24.

CHOUBERT, G.; BLANC, J.M. 1993. Muscle pigmentation changes during and after spawning in male and female rainbow trout, Oncorhynchus mykiss, fed dietary carotenoids. Aquatic Living Resources 6:163-168.

DUTCOSKY, S. D. Análise sensorial de alimentos. Curitiba: Champagnat, 1996.

FARMER, L. J.; MCCONNELL, J.M.; KILPATRICK, D.J. 2000. Sensory characteristics of farmed and wild Atlantic salmon. Aquaculture 187:105-125.

JOHNSTON, I.A.; LI, X.; VIEIRA,V.L.A.;NICKELL, D.; DINGWALL, A.; ALDERSON, R.; CAMPBELL, P.; BICKERDIKE, R. 2006. Muscle and flesh quality traits in wild and farmed Atlantic salmon. Aquaculture 256:323-336.

KUBITZA, F. 2000. Tilápia: tecnologia e planejamento na produção comercial. Degaspari. São Paulo.

LIMA, F.C.T. 2003. Subfamily Bryconinae. Págs 174-181. Em: Reis, R.E.; Kullander, S.O.; Ferraris, C.J. (eds.). Check List of the Freshwater Fishes of South and Central America. Edipucrs. Porto Alegre, Brasil.

MATO GROSSO. Lei No 9.096, de 16 de janeiro de 2009. Diário Oficial 16.01.09 e D.O. 11.03.09. Dispõe sobre a Política da Pesca no Estado de Mato Grosso e dá outras providências. Palácio Paiaguás, em Cuiabá, 16 de janeiro de 2009.

MATOS, L.S. 2014. Dieta, composição química, contaminação por metais pesados e análise sensorial do peixe matrinxã (Brycon falcatus, Müllere Troschel, 1844) em rios Amazônicos. Sinop. Dissertação de Mestrado em Ciências Ambientais. Universidade Federal de Mato Grosso. Brasil.

MULLER J. ; TROSCHEL F.H. 1844. Synopsis generum et specierum familiae characinorum (Prodomus descriptionis novorum generum et specierum). Archiv. Naturgesch 10 (1):81-99.

OGAWA, N.B.P.; MAIA, E.L. 1999 Manual de Pesca: ciência e tecnologia do pescado. Varela. São Paulo, Brasil.

RIBEIRO, S.C.A. TOBINAGA, S. 2002. Avaliação sensorial de filés de matrinchã (Brycon cephalus) processados por métodos combinados. Revista Brasileira de Produtos Agroindustriais, Campina Grande, v.4, n.2, p.101-106.

ROESSLER, E.B.; PANGBORN, R.M.; SIDEL, J.L.; STONE, H. 1978. Expanded statistical tables for estimating significance in paired-preference, paired difference, duo-trio and triangle tests. Journal of Food Science 43 (3):940-943.

SANTAMARIA, F.M.; ANTUNES, S.A. 1998/1999. Coloração e rendimeto do filé de piracanjuba (Brycon orbignyanus, Valenciennes, 1849), (Pisces, Characidae) silvestre e criada em cativeiro. Boletim do Instituto de Pesca 25(único):27-30.

SILVA, R. S., AlMEIDA, F. O., PRATES, G. V. S., CARVALHO, G. B., FERRAZ, D. 2013. Análise do sabor de Tambatinga cultivado em diferentes sistemas de cultivo na região da Transamazônica - Pará. Revista Agrotecnologia, Anápolis 4 (1):74-81.

SYLVIA, G.; MORRISSEY, M.T.; GRAHAM, T.; GARCIA, S. 1995. Organoleptic qualities of farmed and wild salmon. Journal of Aquatic Food Product Technology 4 (1):51-64.

THOMASSEN, M.S.; ROSJO, C. 1989. Different fats in feed for salmon: influence on sensory parameters, growth rate and fatty acids in muscle and heart. Aquaculture 79:129-135.

VIDAL, L.V.O.; ALBINATI, R.C.B.; ALBINATI, A.C.L.; LIRA, A.D.; ALMEIDA T.R.; SANTOS, G.B. 2008. Eugenol como anestésico para a tilápia do Nilo. Pesquisa Agropecuária Brasileira 43 (8):1069-1074. 